Original Research Paper

\title{
Molecular Identification of Leishmania Species in Patient with Cutaneous Leishmaniasis in an Endemic Area of Fars Province, Southern Iran
}

\author{
${ }^{1}$ Vahid Rahmanian, ${ }^{* 1}$ Nader Sharifi, ${ }^{2}$ Zahra Kargar Jahromi, ${ }^{1}$ Hayedeh Parvin, \\ ${ }^{1}$ Narges Rahmanian and ${ }^{3}$ Hekmatollah Khoubfekr \\ ${ }^{I}$ Zoonoses Research Center, Jahrom University of Medical Sciences, Jahrom, Iran \\ ${ }^{2}$ Central Research Laboratory, Jahrom University of Medical Sciences, Jahrom, Iran \\ ${ }^{3}$ Iranshahr Health Services, Iranshahr University of Medical Sciences, Iranshahr, Iran
}

\author{
Article history \\ Received: 30-01-2021 \\ Revised: 08-03-2021 \\ Accepted: 11-03-2021 \\ Corresponding Author: \\ Nader Sharifi \\ Zoonoses Research center, \\ Jahrom University of Medical \\ Sciences, Jahrom, Iran \\ Email: nadersharifi81@yahoo.com
}

\begin{abstract}
The Cutaneous Leishmaniasis (CL) contains a range of factors, which provide different patterns of transmission that make it difficult to control. The aim of this study was to identify the different species of Leishmania in the Jahrom district of Fars province, southern Iran. This descriptive study was done on CL suspected cases who were referential to the Reference of Jahrom Health Center laboratory in 2017 from all different zones mentioned. Demographic variables (age, sex, location, number and place of wounds) was recorded by a questionnaire. The blood smears were checked for the existence of amastigotes by light microscopy. After microscopic examination smears were extract DNA and used in the Polymerase Chain Reaction (PCR) method. Totally 165 slide samples checked were found to be positive for amastigotes via microscopy and positive for leishmanial DNA in the PCR. two (1.2\%) of the slide samples gave the 750-bp indicative of Leishmania tropica and 163 samples $(98.8 \%)$ gave the 560-bp indicative of Leishmania major. Although the actually that both species of CL are common in Iran, but our results showed that in Jahrom district the main specie was L. major. Thus, this fact displays the importance of reservoir rodents in programs of CL control in this County.
\end{abstract}

Keywords: Species Identification, L. Major, L. Tropica, PCR, Iran

\section{Introduction}

Leishmaniasis is a neglected of vector-borne diseases produced by many Leishmania species which can infect both humans and other mammalian animals. Leishmaniasis is can be seen in three main forms such as cutaneous, mucosal and visceral (Rahmanian et al., 2021).

It is estimated that 350 million population, are at risk of Cutaneous leishmaniasis (CL) and 1 million patient have been described in the past five years (Laurent et al., 2017).

In Islamic republic of Iran, two forms of CL are occurs; Zoonotic Cutaneous Leishmaniasis (ZCL) caused by Leishmania major (L. major) and the rodents including Rhombomys opimus, Meriones libycus and Meriones nesokia are considered as the main reservoir (Shirzadi et al., 2015; Mohammadiha et al., 2018). The Anthroponotic Cutaneous Leishmaniasis (ACL) caused by Leishmania tropica (L. tropica) whose main reservoir is human and it is considered to be an excessive significance in many regions of Iran, including some big and middle sized cities such as Mashhad, Tehran, Sabzevar and Neishabur in the north-east, Fars in the south, Kerman and Bam in the southeast, Kashan, Yazd and parts of the city of Esfahan in the central region (Yaghoobi-Ershadi et al., 2013; 2015). Atypical mucosal infections with leishmanial parasites have also been detected in Iran, although infrequently.

Approximately 20,000 cases of CL are reported yearly from provinces of Iran, but it is estimated that the number of actual cases has doubled (Karami et al., 2013; Moein et al., 2018). CL is endemic in 15 provinces of IR and in the recent years. In addition, in recent years, climatic and environmental variables have created suitable conditions for the activity of reservoirs and vectors of CL in some new foci (Moein et al., 2018; Shirzadi et al., 2015). 
Epidemiological studies, taxonomic and population genetics surveys, measuring a specific chemotherapeutic treatment, correct identification of the parasite species and the investigation of the genetic variation of the parasites are necessary for leishmaniasis control program in endemic regions (Leite et al., 2010; Van der Auwera et al., 2013).

In Iran, diagnosis commonly depends on detection of the species in the Giemsa-stained smear that is ready with the aspiration material of lesion. Furthermore, culture in Novy-MacNealNicolle (NNN) medium, ELISA, IFA and histopathological examination are used for investigation. On the other hand, diagnostic ways based on Deoxyribonucleic Acid (DNA) have, nevertheless, now made the sensitive and quick recognition of many germs potential. In recent years, for instance, analyses based on PCR have showed to be valid tools for the identification of Leishmania, without the need to culture the organisms (Mohammadiha et al., 2013).

Fars Province is an endemic cutaneous and visceral leishmaniasis, so that the cumulative incidence of cutaneous and visceral leishmaniasis was reported to be between 59.9-98.8 and 0.58-0.73 cases per 100000 inhabitants, respectively (Shirzadi et al., 2015). The National Leishmaniasis Control Program in Iran due to differences in reservoir, differences in the methods of control and prevention of Leishmania species highlights the essential of determining molecular identification of species in endemic regions once every five years. On the other hand, (Rahmanian et al., 2018), showed in a one study an increasing trend in number of CL cases in Jahrom county, 90 cases in 2010 to 309 cases in 2014 and due to the lack of information about the kinds of species of CL in the county, this study was to identify the different species of Leishmania in the Jahrom district of Fars province, southern Iran.

\section{Methodology}

\section{Study Site}

The Fars province is situated in southern Iran and has a distinct of $122,400 \mathrm{~km}^{2}$. The climate is moderately dusty and dry. The study has been carried out in Jahrom of Fars province. This districtis located at $\sim 53^{\circ} 33^{\prime} \mathrm{E}$, $28^{\circ} 30^{\prime} \mathrm{N}$ and $1050 \mathrm{~m}$ above the sea level. Due to the appropriate ecological situations for leishmaniasis (reservoir hosts, vectors variety and weather conditions), Jahrom has permanently been considered as one of the Cutaneous and Visceral leishmaniasis (CL, VL) and focus district (Davami et al., 2011), (Fig. 1).

\section{Study Design and Sampling Method}

In this descriptive study, sampling lasted in a laboratory for 12 months from January to December 2017 in Jahrom leishmaniasis national surveillance system. The inclusion criteria for entering the study were all patients referred by the physician (imported or autochthonous cases) to the reference laboratory in the surveillance system for the diagnosis of leishmaniasis.

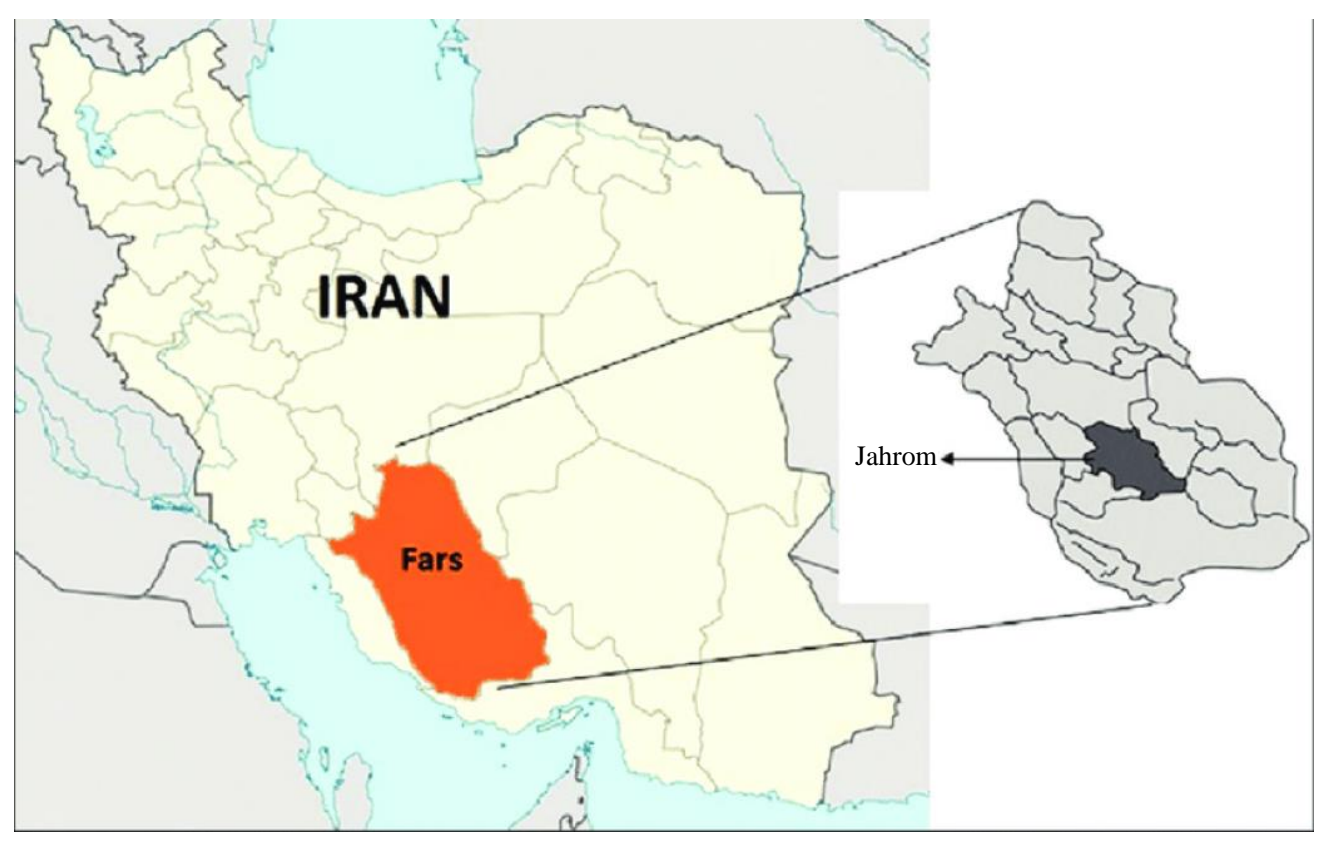

Fig. 1: The location of Jahrom County in Fars province, Iran 
First, infection foci were determined according to the cumulative incidence of the previous year. Then, the samples were aseptically taken from all individuals with suspected CL lesions and referred to the laboratory by the physician after completing the survey form. Two slides were prepared from each of the 165 suspected patients and stained with Giemsa stain. The samples were taken by scalpel from the deep wound through harvesting the tissue containing macrophages, stained with Giemsa stain and explored with a magnification of $\times 100$ to observe the parasite. The surface of positive the samples was scraped from the slides and used for PCR.

\section{DNA Extraction}

The DNA extraction was made via phenolchloroform and proteinase K (Motazedian et al., 2002). Thus, the stained sample on the slide surface was poured into $1.5 \mathrm{mM}$ plus $200 \mu \mathrm{L}$ of lysis buffer and $5 \mu \mathrm{L}$ of proteinase $\mathrm{K}$ and incubated at $56^{\circ} \mathrm{C}$ for $2 \mathrm{~h}$. Then, the contents of the microtube were mixed with $100 \mu \mathrm{L}$ of the phenol/chloroform/isoamyl-alcohol mixture and centrifuged at 15,000 rpm for $5 \mathrm{~min}$. At this stage, three layers were observed; the supernatant containing DNA, the middle layer containing lipids and proteins and the bottom layer containing phenol/chloroform/isoamylalcohol mixture. The supernatant (about 100-150 $\mu \mathrm{L}$ ) was carefully separated and poured into a new microtube and added with about $200-300 \mu \mathrm{L}$ of isopropenol and $10-15 \mu \mathrm{L}$ of $3 \mathrm{M}$ sodium acetate. The samples after mixing in the microtubs were frozen at $-20^{\circ} \mathrm{C}$ for at least $40 \mathrm{~min}$. After defreezing, the samples were centrifuged for $15 \mathrm{~min}$ at $12000 \mathrm{rpm}$. The supernatant was discarded, the resulting precipitate was dried and mixed with $50 \mu \mathrm{L}$ of distilled water and used for the PCR process.

\section{PCR Process}

$\begin{array}{crr}\text { The primers } & \text { of } & \text { CSB1XR } \\ \text { (ATTTTTCGCGATTTTCGCA } & \text { GAACG) and }\end{array}$
CSB2XF (CGAGTAGCAGAAACTCCCGTTCA) were used in the first step and the primers of $13 \mathrm{Z}$ (ACTGGGGGTTGGTGTAAATAG) and LIR (TCGCAGAACGCCCCT) (The products of Cinna Gen Company)(Davami et al., 2011) in the second step. In the first step, each $25 \mu \mathrm{L}$ of the reaction solution contained $5 \mu \mathrm{L}$ of DNA (with a concentration of $200 \mathrm{ng}$ ), $1.5 \mathrm{mM}$ of magnesium chloride, $0.2 \mathrm{mM}$ of deoxynucleotide triphosphates (dNTP mix), $2.5 \mu \mathrm{L}$ PCR buffer (10x), one unit of Taq DNA polymerase and $10 \mu \mathrm{M}$ of CSB1XR and CSB2XF primers (The products of CinnaGen Company). The program used in the thermo cycler (Master cycler gradient; Eppendorf AG, Hamburg, Germany) was as follows: Denaturation temperature of $90^{\circ} \mathrm{C}$ for $5 \mathrm{~min}$, followed by 30 cycles including $90^{\circ} \mathrm{C}$ for $45 \mathrm{sec}, 55^{\circ} \mathrm{C}$ for $1 \mathrm{~min}$ and $72^{\circ} \mathrm{C}$ for $1.5 \mathrm{~min}$ and then $72^{\circ} \mathrm{C}$ for $5 \mathrm{~min}$ (Davami et al., 2014).

The produce of this step of the PCR was diluted with pure water. Then, $1 \mu \mathrm{L}$ of this diluted solution was used in the second step of PCR. All steps are like the first step of PCR, except that the primers of LIR and $13 \mathrm{Z}$ (ACTGGGGGTTGGTGTAAAATAG) were used instead of the primers of the first step and the final volume of the reaction solution increased to $100 \mu \mathrm{L}$ (Davami et al., 2011; Azizi et al., 2013).

Next, $5 \mu \mathrm{L}$ of the sample of the second PCR product was poured into the wells onto $1.5 \%$ agarose gel marked with ethidium bromide for the implementation of electrophoresis and then explored by UV light (Davami et al., 2011).

The size of each observed product was compared with the ladder marker and the positive control sample. The positive controls were tested using DNA extracted from reference promastigote cultures of parasite $L$. major (MHOM/IR/54/LV39) and $L$. tropica (MHOM/IR/89/ARA2), created on any gel (Pourmohammadi et al., 2010).

\section{Results}

165 slide samples checked were positive for amastigotes via microscopy and positive for leishmanial DNA in the PCR. 2(1.2\%) of the slide samples presented the 750-bp second-round amplicon indicative of $L$. tropica and 163 samples $(98.8 \%)$ presented the 560-bp indicative of L. major (Fig. 2).

The average age of the patients was $23.36 \pm 16.13$ (rang,1-87) years. The male to female ratio was 1.4. However, according to Fig. 4, the peak occurrence of CL was documented in October and January and the lowest was documented in May (Fig. 3).

In total, $114(69.09 \%)$ of the patients were villagers and the rest lived in the urban regions.

The average diameter of the wounds was $1.83 \pm 1.37 \mathrm{~cm}$. Hands and face were the most affected body parts with 65 and 38 cases, respectively (Fig. 4).

When we interviewed the cases recognized with $L$. tropica, it was indicated that the patient had previously travelled to an endemic area of ACL in the past year. It has been observed that the patient had one lesion on his right hand and two lesions in the face. It has been considered that the cases can be assessed as an imported case since he has come from Marvdasht in Fars province, lately.

It has been perceived that the second case who has been living in Ghaleini rural had four lesions on his face. Since this case had no travel history to an endemic ACL district, it has been assessed as a native case. 


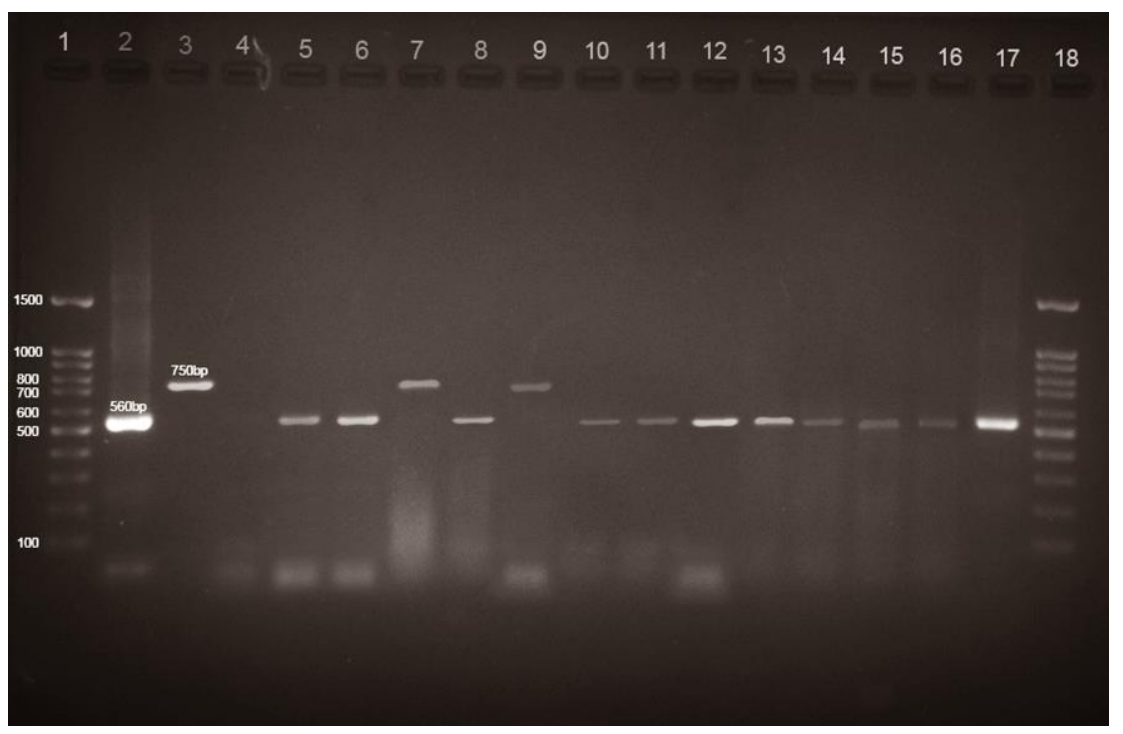

Fig. 2: PCR test results of samples isolated from Jahrom patient's foci. The molecular-weight 'marker' (lane 1), The $L$. major positive control (lane 2) and The L. tropica positive control (lane 3) and test samples identified as L. tropica (lane 7,9) and $L$. major (lanes 5, 6, 8 and 10-17).

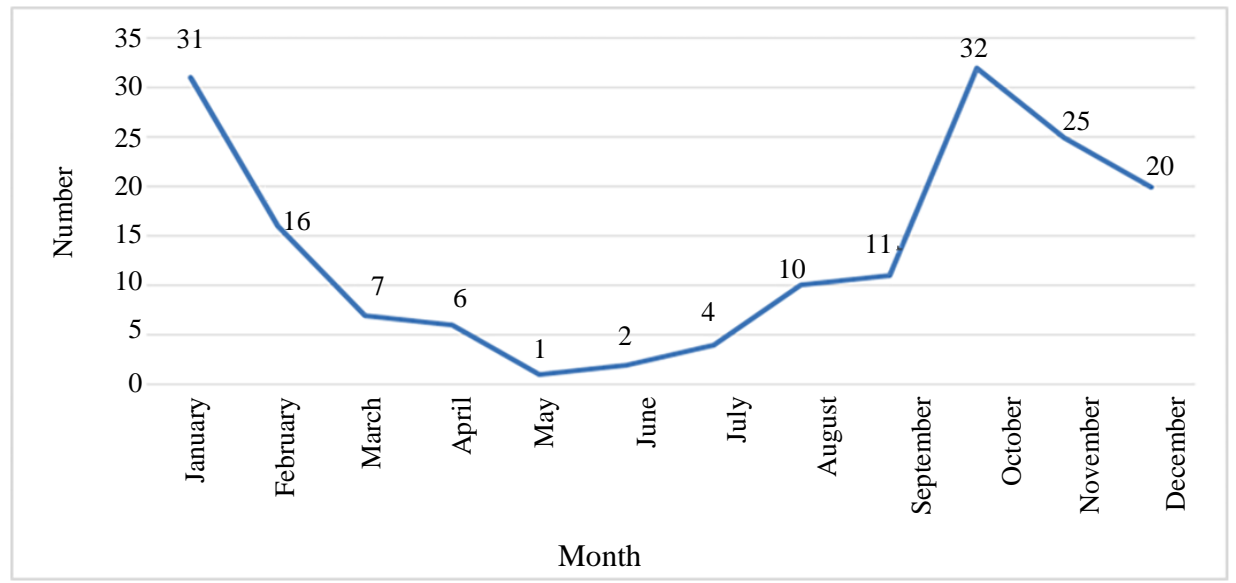

Fig. 3: Frequency of patients with CL in term of months

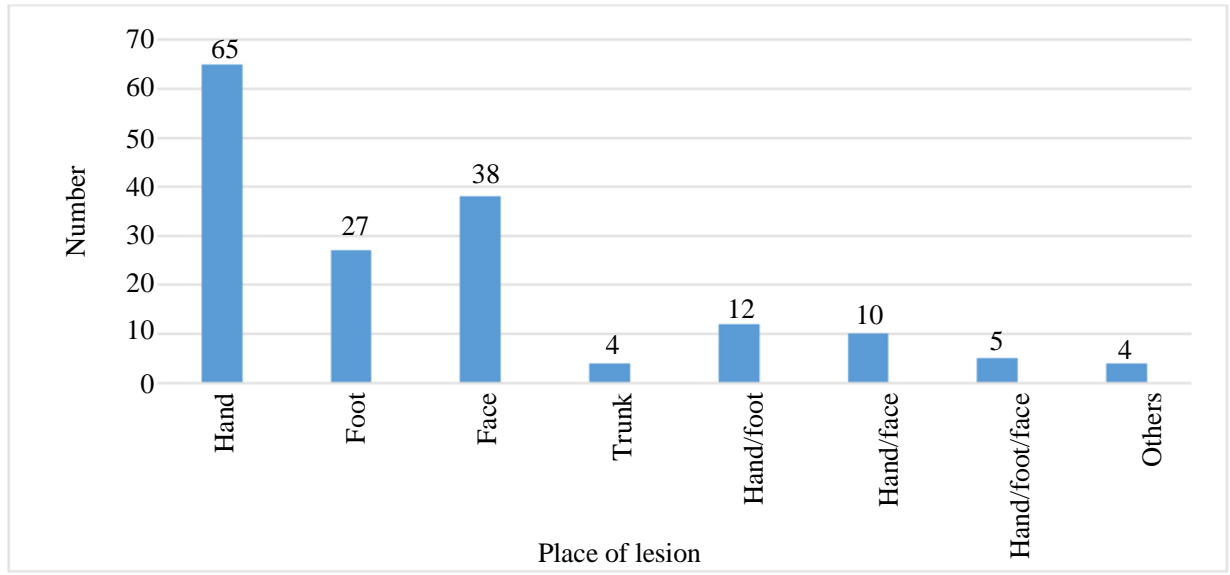

Fig. 4: Frequency of cases with CL in term of place lesion 


\section{Discussion}

Before the emergence of new methods, the identification and isolation of parasites were difficult. Clinical signs, epidemiology of the disease, carriers' examination, ability to cause disease in laboratory animals and growth in the culture medium were factors that were used to detect parasitic species. But nowadays, recognizing genes and using molecular diagnostic methods and using the DNA of organisms, it is easy to determine the species of the parasite (Ghasemian et al., 2011).

In this study, L. majorwas found to be responsible for CL in Jahrom County. It seems that, in 2017, L. major was the cause of practically all the CL in Jahrom with $L$. tropica-attributable disease existing but comparatively infrequent. Thus, specific control programs for this specie of Leishmania is important including rodents elimination as the main reservoir of L. major in Iran, however, this program should be prioritized as it is one of the most important strategic ways to control and prevent this disease.

Detecting the Leishmania species in each endemic area, can be essential for control and prevention program in disease surveillance system (Alvar et al., 2012).

Hatam et al. (2009), examined a number of patients with leishmaniasis in Fars province using iso-enzyme electrophoresis and PCR methods. The finding of this study showed that, all patients were diagnosed with $L$. major and this suggests an increasing in the spread of this type of leishmania in the Fars province.

Previous studies have identified Phlebotomus papatasi and Phlebotomus salehi as a vector of $L$. major and wild rodents and dogs serve as the reservoir of infection in Jahrom district. On the other hand, there are important sandflies such as $P$. Sergentomyiasintoni, $S$. theodori, S. palestinensis, S. baghdadis, S. clydei, S. squamipleuris, $S$. tiberiadis and $S$. antennata, which are considered as potential vectors of leishmaniasis, have been proven in this district (Davami et al., 2011).

Other finding showed that the place of residence, $69.09 \%$ of the patients were rural, which could be a good habitant for the native vectors and reservoir hosts of L. major.

In the present study, both genders were vulnerable to the CL. However, the frequency of CL cases was more prevalent in males than females. Other research from, Fars and Kermanshah Province, stated similar results (Norouzinezhad et al., 2016; Fattahi Bafghi et al., 2016). High incidence of CL in males could be due to various factors such as behaviors of wearing clothes, occupational, travel to endemic areas, poor health literacy, ecological and vector associated variables.

Due to the seasonal orientation, we found that the peak prevalence happened in autumn and winter. This finding is similar with studies in Esfahan, Yazd, Kermanshah and Qom Provinces (Karami et al., 2013;
Yaghoobi-Ershadi et al., 2015; Hamzavi and Khademi, 2015). Increase the number of CL patients in autumn and winter can be related by the incubation period of the CL, weather conditions, sand-fly highest activities and blood sucking of the victims. Therefore, the major control activities of any Leishmania species such as control reservoir, increasing the awareness of the public at risk about the transmission routes and preventive approaches should occur before peak disease incidence.

The CL lesions can occur in any part of the body, however, there are different reports about unusual sites (Doroodgar et al., 2017a-b). In this study, the most common infected sites were hands and face. Our results are similar to the results of some other studies in Iran (Yaghoobi-Ershadi et al., 2015; Norouzinezhad et al., 2016; Karami et al., 2013; Rahmanian et al., 2018).

Since hands are often uncoated and simply exposed to infected sand-fly bites, hands and faces show a higher occurrence of wounds than other parts of the body. Furthermore, sandflies have a short proboscis; therefore, they cannot bite through dresses.

\section{Conclusion}

The samples of leishmaniasis obtained at Jahrom County are mainly of $L$. major, in which the reservoir of wild rodents, especially rats. Therefore, in order to control and prevent CL, rodent control program as one of the most important prevention strategies should be prioritized in this County.

\section{Acknowledgement}

The authors thank the Vice Chancellor for Research of Jahrom University of Medical Sciences for approving and financially supporting this project. Furthermore, we would like to thank health deputy of Jahrom University of Medical Sciences for trying to control these infections and perform primary health care.

\section{Authors' Contributions}

Vahid Rahmanian: Organized the study, proposal of study writing, data gathering, manuscript preparation, data analysis and interpretation of data, literature search and final revision of the study.

Nader Sharifi: Analysis and interpretation of data, manuscript editing, final revision of the study content, final approval of the revision the manuscript.

Zahra Kargar: Design of study, DNA extraction and PCR process, interpretation of data and final approval of the version the manuscript.

Hayedeh Parvin: Data gathering, Design of study, literature search and final approval of the version of the manuscript. 
Narges Rahmanian and Hekmatollah

Khoubfekr: Manuscript editing, literature search, analysis and interpretation of data, final approval of the version of the manuscript.

\section{Ethical Considerations}

The protocol of this study was approved by the Ethics Committee of Jahrom University of Medical Sciences (Cod: IR.JUMS.REC.1395.051).

\section{References}

Alvar, J., Vélez, I. D., Bern, C., Herrero, M., Desjeux, P., Cano, J., ... \& WHO Leishmaniasis Control Team. (2012). Leishmaniasis worldwide and global estimates of its incidence. PloS One, 7(5), e35671. https://doi.org/10.1371/journal.pone.0035671

Azizi, K., Badzohreh, A., Sarkari, B., Fakoorziba, M. R., Kalantari, M., Moemenbellah-Fard, M. D., \& AliAkbarpour, M. (2013). Nested polymerase chain reaction and sequence-based detection of Leishmania infection of sand flies in recently emerged endemic focus of zoonotic cutaneous leishmaniasis, southern Iran. Iranian Journal of Medical Sciences, $38(2$ Suppl), 156. https://www.ncbi.nlm.nih.gov/pmc/articles/PMC3 771217/

Fattahi Bafghi, A., Eftekhari, M., Ahmadi, S., \& Moghateli, M. (2016). Epidemiological pattern of cutaneous leishmaniasis in the province of Fars, Iran (since 2010-2014). Journal of Community Health Research, 5(4), 240-248. http://jhr.ssu.ac.ir/browse.php?a_id=333\&slc_lang= en\&sid $=1 \& \mathrm{ftxt}=0$

Davami, M. H., Motazedian, M. H., Kalantari, M., Asgari, Q., Badzohre, A., \& Mohammadpour, I. (2011). First microscopical and molecular-based characterization of Leishmania major within naturally infected Phlebotomus salehi (Diptera; Psychodidae) in Fars province, southern Iran. Annals of Tropical Medicine \& Parasitology, 105(7), 485-491. https://www.tandfonline.com/doi/abs/10.1179/136485 9411Y.0000000037

Davami, M. H., Motazedian, M. H., Kalantari, M., Asgari, Q., Mohammadpour, I., Sotoodeh-Jahromi, A.,... \& Pourahmad, M. (2014). Molecular survey on detection of Leishmania infection in rodent reservoirs in Jahrom District, Southern Iran. Journal of Arthropod-Borne Diseases, 8(2), 139. https://www.ncbi.nlm.nih.gov/pmc/articles/PMC4 478425/

Doroodgar, M., Doroodgar, M., \& Doroodgar, A. (2017a). Eyelid cutaneous leishmaniasis: a case report. Iranian Journal of Public Health, 46(2), 261. https://doi.org/10.1155/2017/3198547
Doroodgar, M., Doroodgar, M., \& Doroodgar, A. (2017b). Unusual presentation of cutaneous leishmaniasis: ocular leishmaniasis. Case Reports in Infectious Diseases, 2017. https://doi.org/10.1155/2017/3198547

Ghasemian, M., Maraghi, S., Samarbafzadeh, A. R., Jelowdar, A., \& Kalantari, M. (2011). The PCR-based detection and identification of the parasites causing human cutaneous leishmaniasis in the Iranian city of Ahvaz. Annals of Tropical Medicine \& Parasitology, 105(3), 209-215 https://doi.org/10.1179/136485911X12899838683520

Hamzavi, Y., \& Khademi, N. (2015). Trend of cutaneous leishmaniasis in Kermanshah Province, west of Iran from 1990 to 2012. Iranian Journal of Parasitology, $10(1), \quad 78$. https://www.ncbi.nlm.nih.gov/pmc/articles/PMC4 403543/

Hatam, G. R., Rezanezhad, H., Motazedian, M. H., \& Sarkari, B. (2009). In vitro infectivity of Leishmania major isolated from patients with different clinical forms of cutaneous leishmaniasis and its association with parasite Zymodems.

https://www.sid.ir/en/journal/ViewPaper.aspx?ID $=157850$

Karami, M., Doudi, M., \& Setorki, M. (2013). Assessing epidemiology of cutaneous leishmaniasis in Isfahan, Iran. Journal of Vector Borne Diseases, 50(1), 30. http://www.mrcindia.org/journal/issues/501030.pdf

Laurent, K., Susong, J., Fillman, E., \& Ritchie, S. (2017). Cutaneous leishmaniasis in a saudi arabian soldier stationed in the united states. Military Medicine, 182(7), e1953-e1956. https://doi.org/10.7205/MILMED-D-16-00401

Leite, R. S., de Almeida Ferreira, S., Ituassu, L. T., de Melo, M. N., \& de Andrade, A. S. R. (2010). PCR diagnosis of visceral leishmaniasis in asymptomatic dogs using conjunctival swab samples. Veterinary Parasitology, 170(3-4), 201-206. https://doi.org/10.1016/j.vetpar.2010.02.020

Moein, D., Masoud, D., Saeed, M., \& Abbas, D. (2018). Epidemiological aspects of cutaneous leishmaniasis during 2009-2016 in Kashan city, central Iran. The Korean Journal of Parasitology, 56(1), 21. https://doi.org/10.3347/kjp.2018.56.1.21

Mohammadiha, A., Dalimi, A., Mohebali, M., Sharifi, I., Mahmoudi, M., Mirzaei, A.,... \& Ghorbanzadeh, B. (2018). Molecular identification and phylogenetic classification of Leishmania spp. isolated from human cutaneous leishmaniasis in Iran: A cross-sectional study. Iranian Journal of Parasitology, 13(3), 351. https://www.ncbi.nlm.nih.gov/pmc/articles/PMC6 243174/ 
Mohammadiha, A., Mohebali, M., Haghighi, A., Mahdian, R., Abadi, A. R., Zarei, Z.,... \& Akhoundi, B. (2013). Comparison of real-time PCR and conventional PCR with two DNA targets for detection of Leishmania (Leishmania) infantum infection in human and dog blood samples. Experimental Parasitology, 133(1), 89-94. https://doi.org/10.1016/j.exppara.2012.10.017

Motazedian, H., Karamian, M., Noyes, H. A., \& Ardehali, S. D. N. A. (2002). DNA extraction and amplification of Leishmania from archived, Giemsastained slides, for the diagnosis of cutaneous leishmaniasis by PCR. Annals of Tropical Medicine \& Parasitology, 96(1), 31-34. https://www.tandfonline.com/doi/abs/10.1179/0003 49802125000484

Norouzinezhad, F., Ghaffari, F., Norouzinejad, A., Kaveh, F., \& Gouya, M. M. (2016). Cutaneous leishmaniasis in Iran: results from an epidemiological study in urban and rural provinces. Asian Pacific Journal of Tropical Biomedicine, 6(7), 614-619. https://doi.org/10.1016/j.apjtb.2016.05.005

Pourmohammadi, B., Motazedian, M. H., Hatam, G. R., Kalantari, M., Habibi, P., \& Sarkari, B. (2010). Comparison of three methods for diagnosis of cutaneous leishmaniasis. Iranian Journal of Parasitology, 5(4), 1. https://www.ncbi.nlm.nih.gov/pmc/articles/PMC327 9850/

Rahmanian, V., Bokaie, S., Haghdoost, A., \& Barouni, M. (2021). Predicting cutaneous leishmaniasis using SARIMA and Markov switching models in Isfahan, Iran: A time-series study. Asian Pacific Journal of Tropical Medicine, $14(2), \quad 83$. https://doi.org/10.4103/1995-7645.306739
Rahmanian, V., Rahmanian, K., Sarikhani, Y., Jahromi, A. S., \& Madani, A. (2018). Epidemiology of Cutaneous Leishmaniasis, West South of Iran, 20062014. Journal of Research in Medical and Dental Science, 6(2), 378-383. http://eprints.hums.ac.ir/5899/

Shirzadi, M. R., Esfahania, S. B., Mohebalia, M., Ershadia, M. R. Y., Gharachorlo, F., Razavia, M. R., \& Postigo, J. A. R. (2015). Epidemiological status of leishmaniasis in the Islamic Republic of Iran, 1983-2012. Eastern Mediterranean Health Journal, 21(10). https://doi.org/10.26719/2015.21.10.736

Van der Auwera, G., Maes, I., De Doncker, S., Ravel, C., Cnops, L., Van Esbroeck, M.,... \& Dujardin, J. C. (2013). Heat-shock protein 70 gene sequencing for Leishmania species typing in European tropical infectious disease clinics. Eurosurveillance, 18(30), 20543. https://doi.org/10.2807/15607917.ES2013.18.30.20543

Yaghoobi-Ershadi, M. R., Shahbazi, F., Darvishi, M., Akhavan, A. A., Jafari, R., Khajeian, M.,... \& Heidari, M. (2013). Molecular epidemiological study of cutaneous leishmaniasis in the focus of Bushehr city, southwestern Iran. Journal of Arthropod-Borne Diseases, 7(2), 113. https://www.ncbi.nlm.nih.gov/pmc/articles/PMC387 $5877 /$

Yaghoobi-Ershadi, M. R., Marvi-Moghadam, N., Jafari, R., Akhavan, A. A., Solimani, H., Zahrai-Ramazani, A. R.,... \& Dehghan-Dehnavi, A. R. (2015). Some epidemiological aspects of cutaneous leishmaniasis in a new focus, central Iran. Dermatology Research and Practice, 2015. https://doi.org/10.1155/2015/286408 